OPEN ACCESS

Edited by:

Chunyi Zhi,

City University of Hong Kong,

Hong Kong

Reviewed by:

Junchao Zheng,

Central South University, China

Yan Huang,

Harbin Institute of Technology,

Shenzhen, China

*Correspondence:

Hai Duong Pham

phamduong477@gmail.com

Ming-Fa Lin

mflin@mail.ncku.edu.tw

Specialty section:

This article was submitted to

Energy Materials,

a section of the journal

Frontiers in Materials

Received: 02 June 2020

Accepted: 29 July 2020

Published: 11 September 2020

Citation:

Khuong Dien V, Thi Han N,

Nguyen TDH, Huynh TMD, Pham HD and Lin M-F (2020) Geometric and

Electronic Properties of $\mathrm{Li}_{2} \mathrm{GeO}_{3}$.

Front. Mater. 7:288

doi: 10.3389/fmats.2020.00288

\section{Geometric and Electronic Properties of $\mathrm{Li}_{2} \mathrm{GeO}_{3}$}

\author{
Vo Khuong Dien ${ }^{1}$, Nguyen Thi Han 1,2, Thi Dieu Hien Nguyen ${ }^{1,2}$, Thi My Duyen Huynh', \\ Hai Duong Pham ${ }^{1 *}$ and Ming-Fa Lin ${ }^{1,3 *}$ \\ ${ }^{1}$ Department of Physics, National Cheng Kung University, Tainan, Taiwan, ${ }^{2}$ Department of Chemistry, Thai Nguyen University \\ of Education, Thái Nguyên, Vietnam, ${ }^{3}$ Hierarchical Green Energy Materials, Hi- research Center, National Cheng Kung \\ University, Tainan, Taiwan
}

The 3D ternary $\mathrm{Li}_{2} \mathrm{GeO}_{3}$ compound, which could serve as the electrolyte material in $\mathrm{Li}^{+}$-based batteries, exhibits an unusual lattice symmetry (orthorhombic crystal), band structure, charge density distribution and density of states. The essential properties are fully explored through the first-principles method. In the delicate calculations and analyses, the main features of atom-dominated electronic energy spectrum, space-charge distribution, and atom-/orbital-projected density of states are sufficient to identify the critical multi-orbital hybridizations of the chemical bonds: $2 s-\left(2 p_{x}, 2 p_{y}, 2 p_{z}\right)$ and $\left(4 s, 4 p_{x}, 4 p_{y}, 4 p_{z}\right)-\left(2 s, 2 p_{x}, 2 p_{y}, 2 p_{z}\right)$, respectively, for Li-O and Ge-O. This system possesses a large indirect gap of $\mathrm{Eg}=3.77 \mathrm{eV}$. There exist a lot of significant covalent bonds, with an obvious non-uniformity and anisotropy. In addition, spin-dependent magnetic configurations are completely absent. The theoretical framework could be developed to investigate the important features of anode and cathode materials related to lithium oxide compounds.

Keywords: solid-state electrolytes, DFT, Li2GeO3, lithium batteries, multi-orbital hybridizations

\section{INTRODUCTION}

Increasing demands for storage of electricity from solar and wind energy, mobile electronic devices, electric vehicles promote the development of cost-effective and reliable electrical energy storage (Tarascon and Armand, 2001; Cheng et al., 2008, 2011). Among a large number of possible energy storage technologies such as nickel-cadmium batteries, zinc-manganese batteries, nickel-hydrogen batteries, fuel cells, lead-acid batteries, redox flow batteries, Lithium-ion batteries, etc., the rechargeable Lithium-ion batteries (LIBs) has attracted a great deal of attention due to their high specific energy, wide working temperature range, high operational voltage and a long cycle life (Tarascon and Armand, 2001).

A commercial LIB is composed of a negative (cathode) and positive (anode) electrode separated by an electrolyte (Tarascon and Armand, 2001), in which each component should have a good physical, chemical and material properties, especially for the latter ones to ensure the rapid lithium-ion transmission, is compatible with the electrodes and chemically inert at the same time. Apparently, a drastic change of geometric structures is revealed in the cathode/electrolyte/anode materials. The structural transformations between two-metastable configurations during the battery operation is rather complex and thus very difficult to solve.

In general, the cathode and anode systems of $\mathrm{Li}^{+}$-based batteries belong to a class of solidstate materials, such as the three-dimensional (3D) ternary LiFe/Co/NiO (Sakurai et al., 1997; Liu et al., 2002; Chen et al., 2014) and $\mathrm{Li}_{4} \mathrm{Ti}_{5} \mathrm{O}_{12}$ /graphite compounds (Xiang et al., 2012; Nguyen et al., 2020), respectively, whereas conventional electrolytes belong to liquid states, which come 
with have potential security risks concerning volatilization, flammability and explosion. Recent experimental studies indicated that secondary batteries using inorganic solid electrolytes would be the ultimate batteries to resolve the safety issues (Chen et al., 2016). Moreover, the battery cell design would be simplified with solid electrolytes (Wang et al., 2019). Some candidate systems for solid-state electrolytes are $\mathrm{Li}_{3} \mathrm{OCl}$ (Lu et al., 2016), $\mathrm{Li}_{2} \mathrm{SiO}_{3}$ (Nakagawa et al., 2010; Zulueta et al., 2020), and $\mathrm{Li}_{2} \mathrm{GeO}_{3}$ (Liebert et al., 1976; Furusawa and Enokida, 2011; Xiao et al., 2015; Mokhlesur et al., 2016; Zhao etal., 2019). Among them, the later one has attracted significant research interest due to the following reasons: the $3 \mathrm{D}$ ternary $\mathrm{Li}_{2} \mathrm{GeO}_{3}$ is produced by relatively simple and direct methods and possesses a reliable ionic conductivity $\left[1.5 \times 10^{-5}(\Omega . \mathrm{cm})^{-1}\right]$ (Liebert et al., 1976), which is promising as an alternative for the conventional liquid electrolytes. The ternary $\mathrm{Li}_{2} \mathrm{GeO}_{3}$ compound was also reported as a new $\mathrm{Li}^{+}$superionic conductor exhibiting an excellent electrochemical performance, such as cycle stability, charge capacity $\left(725 \mathrm{mAhg}^{-1}\right)$ and rate capability $\left(810 \mathrm{mAhg}^{-1}\right.$ after 35 cycles) (Mokhlesur et al., 2016). Furthermore, other features, such as the large pyroelectric constants ( 5 times larger than that of tourmaline), high piezoelectric constant (5 times larger than that of $\alpha$-quartz), transparency in the visible region and moderate mechanical impedance, can be used in various pyroelectric, piezoelectric, and acousto-optic devices (Haussuuhl et al., 1982; Yu et al., 1987).

On the theoretical side, a number of studies using the molecular dynamics (MD) and density functional theory (DFT) were investigated on the phase stability (Emly et al., 2013; Zhang et al., 2013; Kaboudvand et al., 2019), defect structure (Lu et al., 2015; Kuganathan et al., 2019), electronic properties (Kaboudvand et al., 2019) and the $\mathrm{Li}^{+}$transport mechanisms (Emly et al., 2013; Lu et al., 2015; Wu et al., 2018; Kuganathan et al., 2019) of the electrodes or electrolyte materials of LIBs. The complicated atomic interactions (multi-orbital hybridizations) are generated from the diversified physical or chemical properties and the complex material structure. Therefore, they significantly affect the parameters that control ion-transport in the solid-state LIBs (Zhao et al., 2020). However, systematic investigations into the interaction of the chemical bonds of $\mathrm{Li}^{+}$-rich 3D ternary compounds as a practical electrolyte for LIBs are still rather limited. Especially, the multi-orbital hybridization that is related to the essential properties of the $\mathrm{Li}_{2} \mathrm{GeO}_{3}$ compound is absent in investigations up to now.

The previous numerical studies based on VASP simulations are sufficient in developing the theoretical framework for understanding the diversified material/physical/chemical phenomena. This framework has been successfully used to conduct systematic investigations of one-dimensional (1D) graphene nanoribbons (Lin et al., 2015b), two-dimensional (2D) graphene/silicene with chemical modifications (Lin et al., 2015a; Tran et al., 2018) and the three-dimensional (3D) ternary $\mathrm{Li}_{4} \mathrm{Ti}_{5} \mathrm{O}_{12}$ compound (Nguyen et al., 2020). Through the delicate analysis, the diversified phenomena of the geometric, electronic and magnetic properties due to different dimensionalities, planar or buckled honeycomb lattices, layer numbers, stacking configurations, adatom chemisorptions, guest-atom substitutions and bulk properties of 3D materials can be fully understood. Therefore, this calculation might be very suitable for investigating the extraordinary properties in a lot of complex oxide compounds, e.g., $\mathrm{Li}_{2} \mathrm{SiO}_{3}, \mathrm{Li}_{2} \mathrm{GeO}_{3}, \mathrm{Li}_{4} \mathrm{Ti}_{5} \mathrm{O}_{12}, \mathrm{LiFe} / \mathrm{Co} / \mathrm{NiO}$, in main-stream $\mathrm{Li}^{+}$-based batteries.

In this paper, the geometric symmetries and electronic properties of the 3D ternary $\mathrm{Li}_{2} \mathrm{GeO}_{3}$ compound (the electrolyte material of $\mathrm{Li}^{+}$-based batteries) are systematically investigated. The state-of-the-art analysis conducted on the various chemical bonds in a large unit cell, the band structure with atomic domination, the atom-/orbital projected density of states (DOS) and the spatial charge density is capable of providing the critical multi-orbital hybridizations. The spin density distribution, the spin-degenerate/spin-split energy bands around the low-energy regions, and the net magnetic moment also be examined in the detail as to whether the magnetism could exist in this compound. The theoretical predictions on the relaxation structure, the valence states, the whole energy spectrum and the band gap could be examined via Powder X-ray Diffraction (PXRD)/Tunneling Electron Microscopy (TEM)/Scanning Electron Microscopy (SEM)/Scanning Tunneling Microscopy (STM), Angle-Resolved Photo Emission Spectroscopy (ARPESS), Scanning Tunneling Spectroscopy (STS) and optical absorption spectra, respectively. In addition, whether a combination of the phenomenological models with the numerical simulations can be achieved in a reliable manner to clarify independent cases is very important, since this linking is for a full exploration of various properties, such as optical, magneto-electronic and transport properties. Therefore, the close relationship between the tight-binding model and the first-principle calculations is thoroughly examined. The present work provides more perceptive insights into the understanding of the diversified chemical bonding, as well as the electronic properties of $\mathrm{Li}_{2} \mathrm{GeO}_{3}$ for the future promising electrolytes of LIBs.

\section{COMPUTATIONAL DETAILS}

We used the density functional theory method via the Vienna $A b$ initio Simmulation Package (VASP) (Kresse and Hafner, 1993) to perform the optimization of crystal structures and the calculation of the electronic properties. The Perdew-Burke-Ernzerhof (PBE) generalized gradient approximation was used for the exchangecorrelation functional (Perdew et al., 1996). The interaction between the valence electrons and ions core was evaluated by the projector augmented wave (PAW) method (Kresse and Joubert, 1999). The cutoff energy for the expansion of the plane wave basis set is $600 \mathrm{eV}$ for all calculation. The Brillouin zone was integrated with a special k-point mesh of $9 \times 9 \times 9$ and $21 \times 21 \times 21$ in the Monkhorst-Pack sampling technique (Wisesa et al., 2016) for the geometric relaxation and electronic calculation, respectively. The convergence condition of the ground-state is set to be $10^{-6}$ $\mathrm{eV}$ between two consecutive simulation steps, and all atoms were allowed to fully relax during the geometry optimization until the Hellmann-Feynman force acting on each atom was smaller than 
A
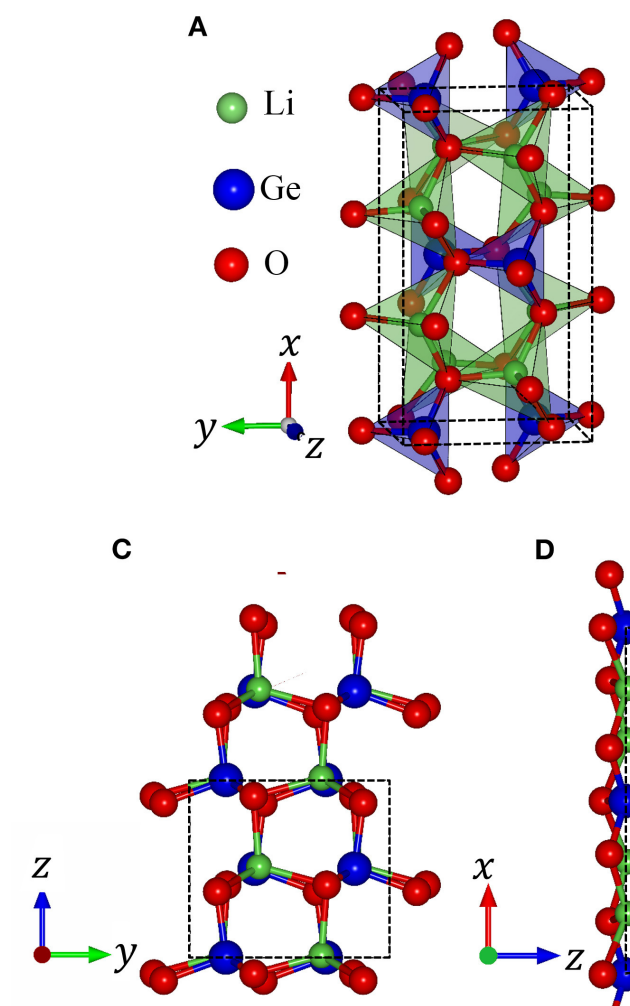

D

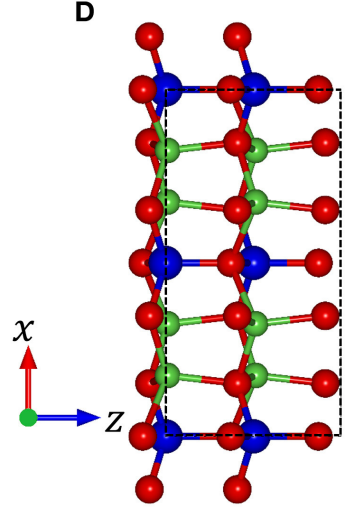

B
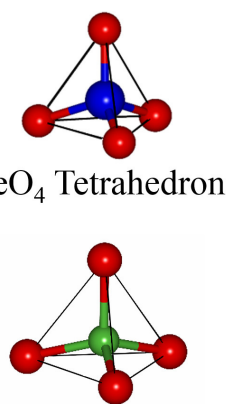

$\mathrm{LiO}_{4}$ Tetrahedron

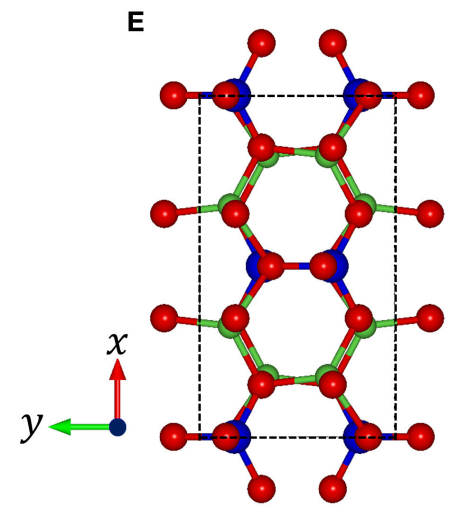

FIGURE 1 | (A) Polyhedron structure of $\mathrm{Li}_{2} \mathrm{GeO}_{3}$, (B) Oxygen (O) coordination around the Lithium (Li), Germanium (Ge) atoms. The geometric structure of $\mathrm{Li}_{2} \mathrm{GeO}_{3}$ along the different projections (C) (100), (D) (010), and (E) (001).

$0.01 \mathrm{eV} / \AA ̊$. Spin-polarized calculations were performed for the geometry optimization and the calculation of the band structure. In the k-point sample, the cutoff energy has been checked for convergence of the calculations.

\section{RESULTS AND DISCUSSIONS}

\subsection{Optimized Structure}

In order to clearly illustrate the complex physical, chemical and material environments in the $3 \mathrm{D}$ ternary $\mathrm{Li}_{2} \mathrm{GeO}_{3}$ compound, one of the meta-stable configurations is chosen. The geometric configuration of $\mathrm{Li}_{2} \mathrm{GeO}_{3}$, as shown in Figure 1, corresponds to the orthorhombic structure with the Cmc21 space group. The conventional cell considered in this work contains 24 atoms $(8 \mathrm{Li}$, $4 \mathrm{Ge}$ and $12 \mathrm{O}$ ), with the lattice parameters being $9.612 \AA, 5.462$ $\AA$, and $4.874 \AA$ for the $x, y$, and $z$ directions, respectively. The basic structural unit is comprised of corner-sharing $\left[\mathrm{GeO}_{4}\right]$ and $\left[\mathrm{LiO}_{4}\right]$ tetrahedra, in which both $\mathrm{Li}$ and $\mathrm{Ge}$ ions are coordinated by four $\mathrm{O}$ ions.

Based on the first-principles calculations for the optimal geometric structure, the $3 \mathrm{D}$ ternary $\mathrm{Li}_{2} \mathrm{GeO}_{3}$ electrolyte material presents unusual crystal lattice symmetries. Obviously, there exists a highly anisotropic and extremely non-uniform structure. The diverse atomic arrangements are easily observed under projections on different planes. e.g., the geometric structures for the (100), (010), and (001) directions (Figures 1C-E), where $\mathrm{Li}, \mathrm{Ge}$, and $\mathrm{O}$ atoms are represented by the green, blue and red balls, respectively. As shown in Table 1, there exist only two kinds of chemical bonds, the Li-O and Ge$\mathrm{O}$ ones, with the total number of bonds being 32 and 16, respectively. According to the delicate first principle calculations, the $\mathrm{Li}_{2} \mathrm{GeO}_{3}$ compound displays modulated bond lengths in the ranges of $\sim 1.930-2.126 \AA$ and $\sim 1.725-1.836 \AA$; the fluctuation percentages $\Delta \mathrm{b}(\%)=\mid$ bond $_{\max }-$ bond $_{\min } \mid /$ bond $_{\min }$ are over 10.1 and $6.4 \%$ for the $\mathrm{Li}-\mathrm{O}$ and $\mathrm{Ge}-\mathrm{O}$ bonds, respectively. The various chemical bonds, which are generated by multi-orbital hybridizations, will directly reflect the distribution width of the spatial charge density (discussed later). Such complicated behaviors might induce extra theoretical barriers (various hopping integrals) in exploring the phenomenological models. Most importantly, the strong fluctuation of bond lengths, which generate an extremely non-uniform environment, is expected to be very important in achieving the outstanding charging and discharging processes (Wang et al., 2015). Due to this, the various intermediate configurations can easily be transformed during the battery operation.

Up until recently, PXRD was the most efficient technique in identifying the lattice symmetries of $3 \mathrm{D}$ materials (Holder and Schaak, 2019). Apparently, it is very suitable to directly observe the $3 \mathrm{D}$ ternary $\mathrm{Li}_{2} \mathrm{GeO}_{3}$ compound. Furthermore, other 
TABLE 1 | Structure parameter of 3D ternary $\mathrm{Li}_{2} \mathrm{GeO}_{3}$ compound.

\begin{tabular}{|c|c|c|c|c|c|c|c|c|c|}
\hline & \multicolumn{3}{|c|}{ Lattice constants } & \multicolumn{4}{|c|}{ Atomic coordinates } & \multicolumn{2}{|c|}{ Bond length } \\
\hline & a (Å) & b (Å) & $c(\AA ̊)$ & Li & $\mathrm{Ge}$ & $O_{1}$ & $\mathrm{O}_{2}$ & Li-O & Ge-O \\
\hline This work & 9.612 & 5.462 & 4.874 & $\begin{array}{l}(0.175 \\
0.342 \\
0.016)\end{array}$ & $\begin{array}{c}(0.0 \\
0.179 \\
0.507)\end{array}$ & $\begin{array}{c}(0.0 \\
0.137 \\
0.880)\end{array}$ & $\begin{array}{l}(0.153 \\
0.318 \\
0.413)\end{array}$ & $\begin{array}{l}1.930(8) \\
1.932(8) \\
1.951(8) \\
2.126(8)\end{array}$ & $\begin{array}{l}1.725(8) \\
1.833(4) \\
1.836(4)\end{array}$ \\
\hline $\begin{array}{l}\text { X-ray (Lee et al., 2008) } \\
\text { diffraction }\end{array}$ & 9.632 & 5.479 & 4.842 & - & - & - & - & - & - \\
\hline $\begin{array}{l}\text { X-ray (Mokhlesur et al., 2016) } \\
\text { diffraction }\end{array}$ & 9.602 & 5.502 & 4.849 & $\begin{array}{l}(0.176 \\
0.344 \\
0.015)\end{array}$ & $\begin{array}{c}(0.0 \\
0.178 \\
0.5)\end{array}$ & $\begin{array}{c}(0 \\
0.190 \\
0.879)\end{array}$ & $\begin{array}{l}(0.162 \\
0.327 \\
0.419)\end{array}$ & - & - \\
\hline
\end{tabular}

The experimental results are also listed.

quantities, such as the particle size and morphology of the sample, can be observed by using SEM (Hansma, 1987). The top view of the nano-materials is observed by using TEM (Feist et al., 2017), while the side view is usually tested by using STM (Carstens et al., 2016). These methods have been successfully utilized to examine the unique geometric structures of graphenerelated systems, such as the multi-walled cylindrical structure of carbon nanotubes (Kelly et al., 1999), folded and scrolled (Zhang et al., 2010; Maitra et al., 2012), profiles of graphene nanoribbons (Van der Lit et al., 2015), as well as stacking configurations and interlayer distances of multi-layer graphene (Lee et al., 2008; Warner et al., 2008). The predicted unusual geometric structure of $\mathrm{Li}_{2} \mathrm{GeO}_{3}$, which includes a non-uniform and the highly anisotropic environment, is in good agreement with the available experimental examinations (Mokhlesur et al., 2016; Yin et al., 2018) (Table 1).

\subsection{Electronic Properties}

The $3 \mathrm{D}$ ternary $\mathrm{Li}_{2} \mathrm{GeO}_{3}$ compound, a $\mathrm{Li}^{+}$-based electrolyte material, exhibits unusual geometric structures and thus diversified electronic properties. The calculated band structure of the $\mathrm{Li}_{2} \mathrm{GeO}_{3}$ compound is presented in Figure 2A. The zeroenergy at the middle of the valence and conduction band is regarded as Fermi energy level. The presence of numerous valence and conduction energy sub-bands in the calculation band structure is a consequence of the many outer orbitals and atoms in the large unit cell. The energy dispersions, which are shown along the high symmetry points, have strongly anisotropic behavior. For example, there exist parabolic, oscillatory and partially flat dispersions. In addition, subband anti-crossing, crossing and non-crossing behaviors come into existence frequently, consequently creating the extremely complicated electronic structure. That is to say, it is very difficult to identify/distinguish/examine the various structures for the different sub-bands, as well as to characterize the width of each energy sub-band. Not only that, but plenty of band-edge states might come into existence in the high-symmetry points. These critical points in the energy-wave-vector space would induce the unique van Hove singularities ( $\mathrm{Li}$ et al., 2010) and thus create the strong absorption structures in the optical properties (Kataura et al., 1999).
In general, the unoccupied states are highly asymmetric to the occupied states about the Fermi level; particularly, the latter have more energy sub-bands and therefore, are the dominating ones. This behavior might be closely related to the complex orbital hybridizations in the $\mathrm{Li}-\mathrm{O}$ and $\mathrm{Ge}-\mathrm{O}$ bonds. Most importantly, the extrema of the conduction band and the valence band are located at the $\Gamma$ and $\mathrm{Z}$ point in the first Brillouin zone, respectively, which thus leads to a large indirect band gap of 3.77 $\mathrm{eV}$. This indicates that the $\mathrm{Li}_{2} \mathrm{GeO}_{3}$ compound has an excellent electrochemical stability and provides a negligible electronic conductivity ( $\mathrm{Wu}$ et al., 2018). However, the investigations for the band structure of the $\mathrm{Li}_{2} \mathrm{GeO}_{3}$ compound are rather limit up to now; therefore, the present results may provide a helpful information for the future work. It is also noted that, under this large band gap, the spin split/degeneracy of the conduction and the valence band near the Fermi level can be not created. Therefore, no magnetic moment exists in this compound, making the spin density distribution meaningless. The mentioned characteristics will be reflected in the density of states.

In addition to the electronic band structure, the atomdominated band structures for valence and conduction states are available to understand the critical chemical bondings. In Figures 2B-D, the contribution to electronic states of $\mathrm{Li}, \mathrm{Ge}$ and $\mathrm{O}$ atoms are denoted by the green, blue and red balls, respectively. The effective energy range related to the $\mathrm{Li}-\mathrm{O}$ and $\mathrm{Ge}-\mathrm{O}$ chemical bonds lies in the range of $-6.5 \mathrm{eV}$ to $6.5 \mathrm{eV}$. It is very hard for the eye to observe the Li-dominations (small radii in Figure 2B) because of only single 2 s orbital takes parks in the chemical bonding. In sharp contrast, it is clearly shown that the $\mathrm{Ge}$ and $\mathrm{O}$ atoms make a significant contribution to the electronic energy spectrum. Especially the latter dominates almost all the occupied and unoccupied bands, and the largest concentration at the top of the valence band (red balls in Figure 2D) since they are related to the entirely of the chemical bonds, with the (2s, $\left.2 \mathrm{p}_{x}, 2 \mathrm{p}_{y}, 2 \mathrm{p}_{z}\right)$-orbital energies. Although the contributions are different, almost all atoms are present in the whole band structure, which may elucidate the large modulation of the Li-O and $\mathrm{Ge}-\mathrm{O}$ chemical bond.

From an experimental aspect, the large band gap due to the extremely strong covalent bonding of the $3 \mathrm{D}$ ternary $\mathrm{Li}_{2} \mathrm{GeO}_{3}$ 

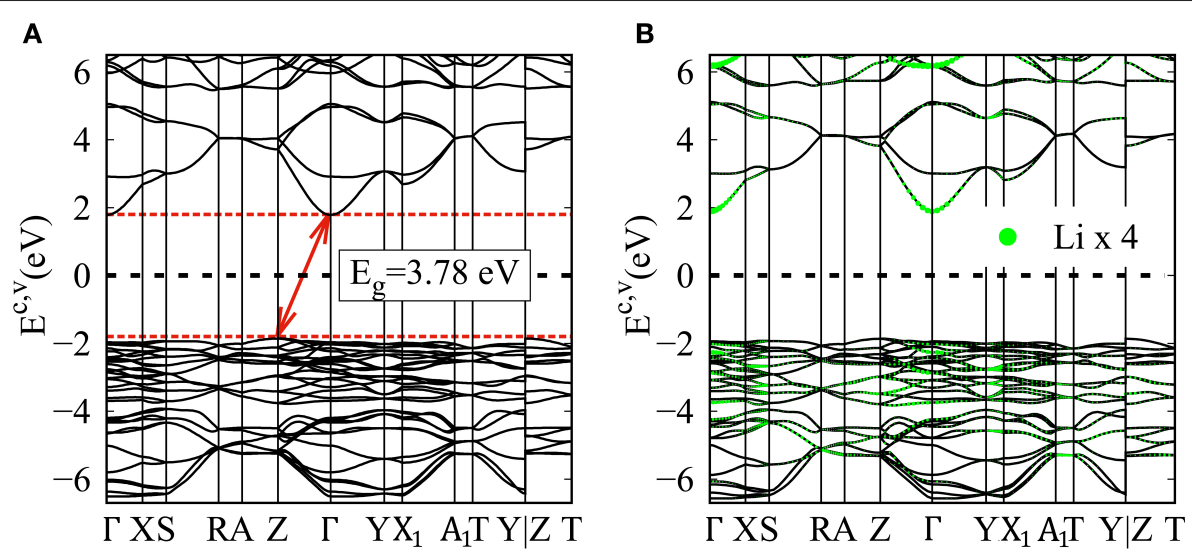

C

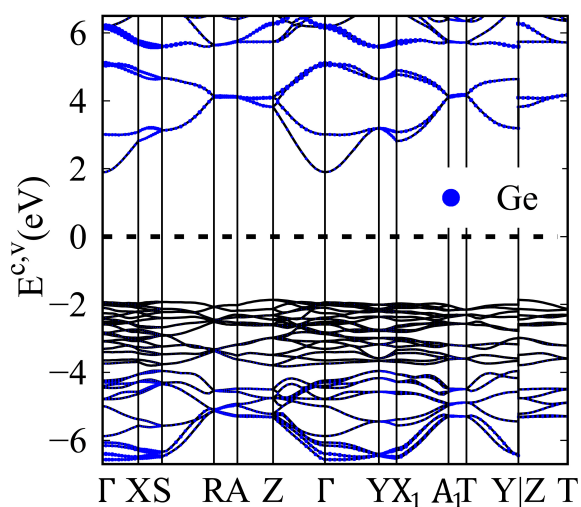

D

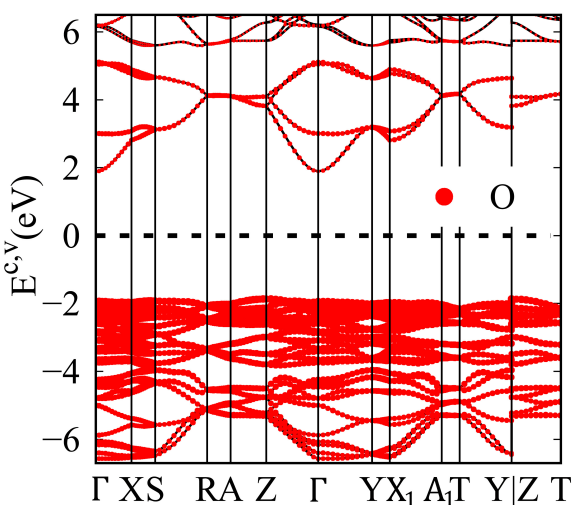

FIGURE 2 | (A) Electronic energy spectrum for the $\mathrm{Li}_{2} \mathrm{GeO}_{3}$ compound, for the specific (B) Lithium (enlarge four times), (C) Germanium, and (D) Oxygen dominances.

compound is directly verified by using optical absorption spectroscopy (Rincon et al., 2003). Apparently, because our system is an indirect gap material, the optical gap should be larger than $3.8 \mathrm{eV}$ as evidenced in previous work (Trukhin et al., 1997). On the other hand, the predictions on the wave-vector-dependent band structures in the occupied states could be examined by ARPES measurements (Lu et al., 2012), which has been utilized in many previous experiments to successfully observe the diversified valence bands in emergent graphene-related materials. For example, the monolayer-like and bilayer-like energy dispersions, respectively, at the $\mathrm{K}$ and $\mathrm{H}$ symmetry points in the stacked graphite (Gruneis et al., 2008). The parabolic/parabolic and linear dispersions in ABstacked bilayer/trilayer graphene (Ohta et al., 2007; Kim et al., 2013), the Sombrero-shaped and partly flat band in ABstacked trilayer graphene (Coletti et al., 2013). The parabolic dispersions in graphene nanoribbons (Balog et al., 2010). Up to now, similar measurement on the unusual energy bands in the $3 \mathrm{D}$ ternary $\mathrm{Li}_{2} \mathrm{GeO}_{3}$ compound is absent. Further examinations are required for the feature-rich and unique energy spectra, including numerous energy subbands, various energy dispersions and the large indirect energy gap. These are useful in understanding the multi-orbital hybridizations of the chemical bonds.

\subsection{Charge Densities and Orbital Hybridizations}

The variation of bond lengths can be formed by the complicated contributions of the available orbitals in different atoms. The spatial charge distributions, $(\rho)$, as indicated in Figures 3a-g, linking the atom-dominated band structure (Figures 2B-D) and the atom-/orbital-projected DOS (later discussions on Figure 5), could provide very useful information on the chemical bondings.

It can be seen that the carrier densities are strongly dependent on the kind of chemical bonds and very sensitive to the modulation of bond lengths. As for the Li-O bonds (Figures 3a-c), the diluted charge density around the Li atom is only contributed by the single 2 s-orbital. Its effective distribution range is approximately $0.5 \AA$, as seen by the extension of the yellow to the green parts of the outermost orbitals. Apparently, the two $1 \mathrm{~s}$ orbitals do not enter into the orbital hybridizations with the $\mathrm{O}$ atom, because it belongs to fully filled electronic configuration. The similar, but wider distribution, which corresponds to the $\mathrm{O}$ case, in which the inner and outer region (the heavy red and green parts) is associated with $2 \mathrm{~s}$ and $\left(2 \mathrm{p}_{x}, 2 \mathrm{p}_{y}, 2 \mathrm{p}_{z}\right)$ ones. However, the O-2s orbitals are relatively far away from the Li-atom and remain in almost the perfect spherical shape. Thus, their contributions to the Li-O chemical bonds are negligible. A weak, but significant overlap of distinct orbitals 

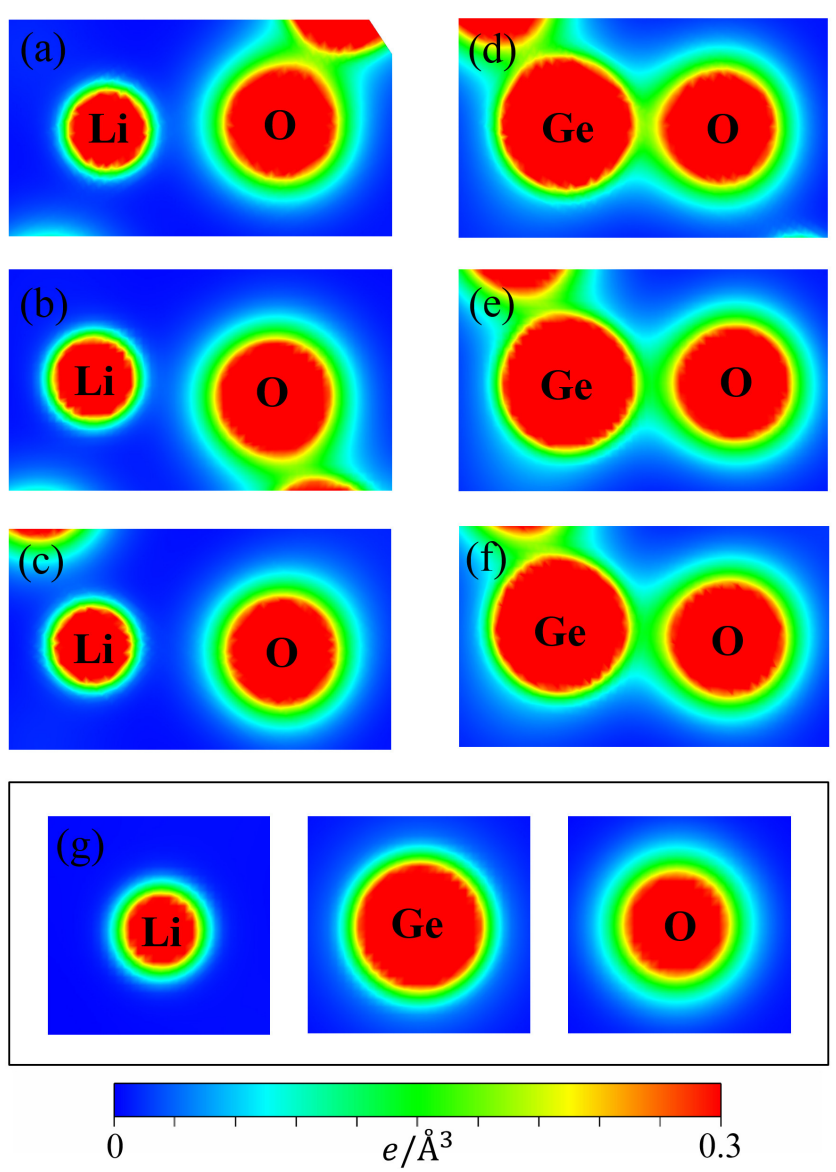

FIGURE 3 | The charge density distribution for the shortest/medium/longest $(\mathbf{a}-\mathbf{c}) \mathrm{Li}-\mathrm{O}$ bonds and $(\mathbf{d}-\mathbf{f}) \mathrm{Ge}-\mathrm{O}$ bonds and $\mathbf{( g )}$ for the isolated (Li, Ge, O) atoms.

between $\mathrm{Li}$ and $\mathrm{O}$ atoms is observed. With the increase of the LiO bond length, this overlap is obviously decreased. As a result, the diverse hopping integrals is presented in the multi-orbital $2 \mathrm{~s}-\left(2 \mathrm{p}_{x}, 2 \mathrm{p}_{y}, 2 \mathrm{p}_{z}\right)$ hybridizations of Li-O bonds.

In striking contrast, the Ge-O bonds, as clearly shown in Figures 3d-f, present very high carrier densities between the $\mathrm{Ge}$ and $\mathrm{O}$ atoms, indicating a rather stronger chemical bonding than the Li-O bonds and thus, explain why the former is shorter than the latter. Furthermore, we can observe that charge density appears around the $\mathrm{Ge}$ atom is larger than that of $\mathrm{Li}$ and $\mathrm{O}$ ones by reason of the larger atomic number. Its effective region (heavy red, blue-green regions) correspond to the $4 \mathrm{~s}$ and ( $4 \mathrm{p}_{x}$, $4 \mathrm{p}_{y}, 4 \mathrm{p}_{z}$ ) orbitals. Very interestingly, the deformed spherical distributions of the spatial charge density between Ge-O bonds clearly illustrate that both $\mathrm{Ge}$ and $\mathrm{O}$ atoms contribute their all valence electrons to form chemical bonds. According to the above-mentioned features, the Ge-O chemical bonds are predicted to exhibit the strong overlap of $\left(4 \mathrm{~s}, 4 \mathrm{p}_{x}, 4 \mathrm{p}_{y}, 4 \mathrm{p}_{z}\right)-(2 \mathrm{~s}$, $\left.2 \mathrm{p}_{x}, 2 \mathrm{p}_{y}, 2 \mathrm{p}_{z}\right)$ orbitals. However, with the bond length increase, the modifications on them are observable, as indicated by the decreased carrier density between the $\mathrm{Ge}$ and $\mathrm{O}$ atoms. The highly non-uniform Li-O and Ge-O chemical bondings might

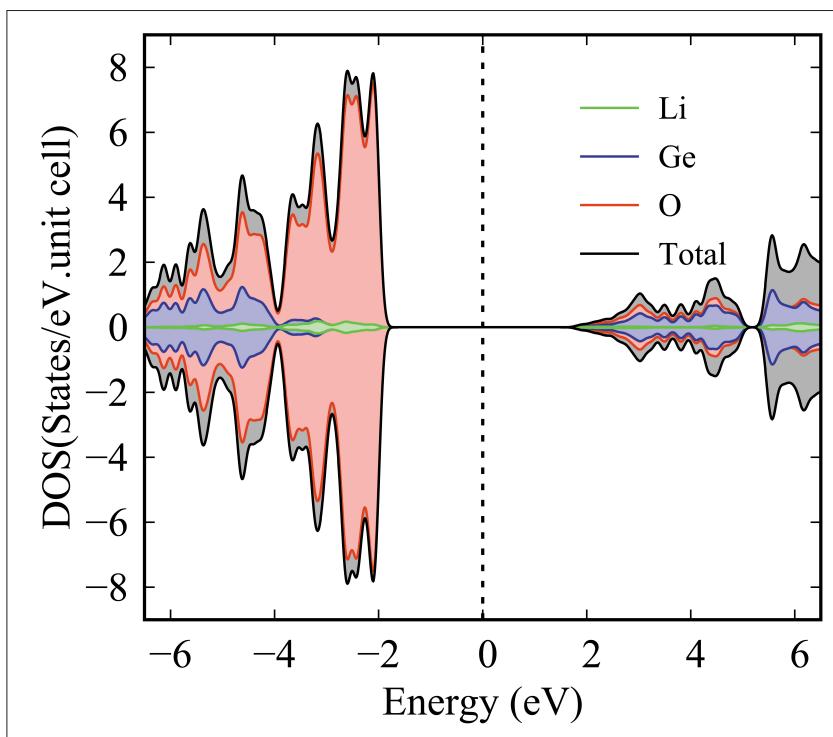

FIGURE 4 | The atom-density of states of $\mathrm{Li}_{2} \mathrm{GeO}_{3}$ compound.

generate numerous difficulties in the phenomenological models (e.g., various hopping integrals).

As mentioned above, the strength of the Ge-O bonds is much stronger than that of the Li-O bonds. The Bader charge analysis of the $\mathrm{Li}_{2} \mathrm{GeO}_{3}$ compound has been done to verify this conclusion. It is found that the average effective charge of $\mathrm{Li}, \mathrm{Ge}$, and $\mathrm{O}$ atoms, respectively, are $0.88 \mathrm{e}, 2.13 \mathrm{e}$, and $-1.3 \mathrm{e}$. This indicates that the $\mathrm{O}$ atom receives the electron from the $\mathrm{Li}$ and $\mathrm{Ge}$ atoms to forms the more stable configuration. Obviously, the effective charge transfer of $\mathrm{Ge}$ attached to the $\mathrm{O}$ is much larger than that of the $\mathrm{Li}$ bridging to the $\mathrm{O}$ and thus, illustrated the former is stronger than the latter ones. It is very important to note that such characteristics are very necessary for the outstanding release and recovery of lithium ions from the electrolyte material during the charging and discharging processes. In other words, the creation of a Li-vacancy is supported by the strong Ge-O bondings and then drives the ion transport.

The density of states (DOS) is characterized as the number of electronic states within a rather small energy range of $\mathrm{dE}$ and directly reflects the primary characteristics of the main features of the valence and conduction energy spectra simultaneously. The atom- and orbital-projected DOS, as shown in Figures 4, 5 , can be used to fully understand the significant multi-orbital hybridizations of $\mathrm{Li}-\mathrm{O}$ and $\mathrm{Ge}-\mathrm{O}$ chemical bonds. As for the Figure 4, the ternary $3 \mathrm{D} \mathrm{Li}_{2} \mathrm{GeO}_{3}$ compound exhibits a lot of special structures (Van Hove singularities), including shoulder structures and asymmetric/symmetric peaks. Such features originate mainly from the saddle, local minimum, maximum and dispersionless relation in the energy band structure. Consistently with the electronic band structure, for a wide gap semiconductor of $3.77 \mathrm{eV}$, the DOS per unit cell of the $\mathrm{Li}_{2} \mathrm{GeO}_{3}$ compound vanishes around the centered Fermi level. This disappearance separates the high asymmetry in holes and electrons in the spectra. As can be seen, most of the atoms dominate in the 


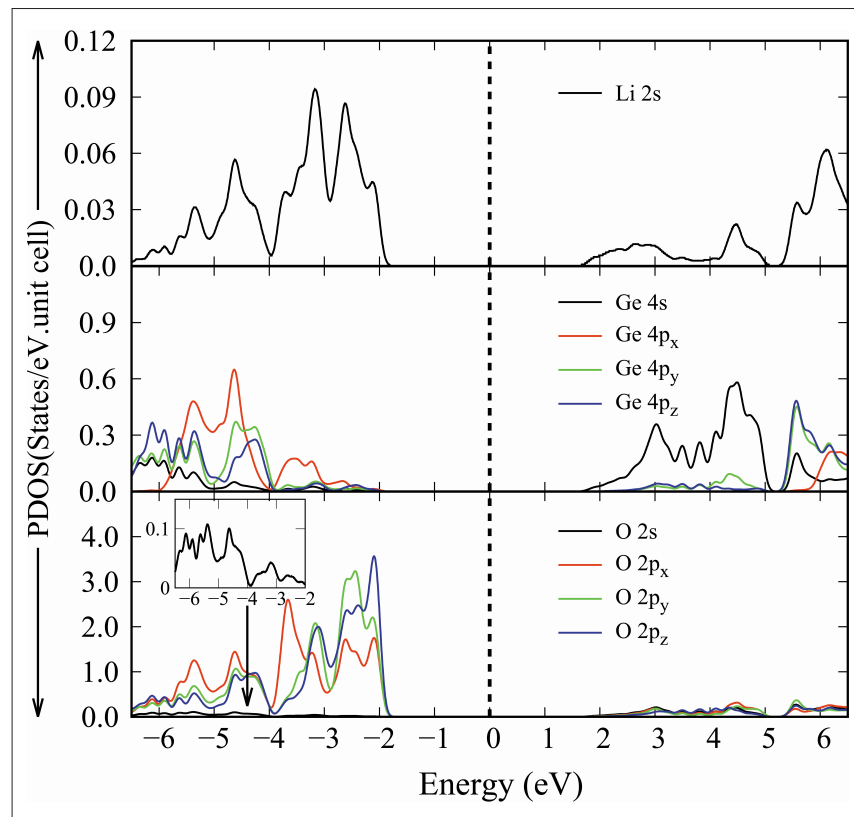

FIGURE 5 | The orbital-projected density of states of $\mathrm{Li}_{2} \mathrm{GeO}_{3}$ compound.

occupied states, but not in the unoccupied ones. The spinup and spin-down DOS of the former and the latter are the same. In them the difference determines the strength of the net magnetic moment, this indicates an absence of magnetism in the system. Furthermore, the three different kinds of atoms have a significantly different contribution in the conduction and valence bands. For example, the most prominent curve, which belongs to the $\mathrm{O}$ atoms, shows a large contribution over the entire energy range, specifically for the top of the valence band. Markedly different, the Li atoms have an almost insignificant contribution, but they are nonetheless very important to create a solid structure of $\mathrm{Li}_{2} \mathrm{GeO}_{3}$. Such a result is mainly defined by the number and available valence orbitals of each atom in a unit cell that takes part in the chemical bondings.

Generally speaking, the various orbitals making an important contribution can be classified into three groups: (I) Li-2s orbital (black curve in the top of Figure 5), (II) Ge-( $\left.4 \mathrm{~s}, 4 \mathrm{p}_{x}, 4 \mathrm{p}_{y}, 4 \mathrm{p}_{z}\right)$ orbitals (black, red, green and blue curves in the middle of Figure 5), and (III) O-(2s, 2 $\left.\mathrm{p}_{x}, 2 \mathrm{p}_{y}, 2 \mathrm{p}_{z}\right)$ orbitals (black, red, blue, and green curves in the lowest of Figure 5). The effective energy spectrum that relates to the $\mathrm{Li}-\mathrm{O}$ and $\mathrm{Ge}-\mathrm{O}$ chemical bonds lie in the range of -6.5 to $6.5 \mathrm{eV}$, as indicated in Figure 5. The delicate analysis of the enlarged orbital-projected DOS clearly provides the number, energy, intensity and form of Li-2s Van hove singularities. Such structures merge well with the $\left(2 \mathrm{p}_{x}\right.$, $2 \mathrm{p}_{y}, 2 \mathrm{p}_{z}$ )-projected DOS for $\mathrm{O}$ atoms, reflecting the importance of the multi-orbital hybridizations, and thus illustrate the large bond length modulation (10.1\%) in the Li-O chemical bonds. However, the contribution the $\mathrm{O}-2 \mathrm{~s}$ orbitals into the specific energy range are negligible. Because they are quite low in energy and thus would not combine efficiently with the Li-2s orbitals. Beside the Li-O bonds, the Ge-O bonds also exhibit a very complicated orbital-hybridization. Concerning the Ge atoms, there exist many strong peaks related to the $\left(4 \mathrm{~s}, 4 \mathrm{p}_{x}, 4 \mathrm{p}_{y}, 4 \mathrm{p}_{z}\right)$ orbitals. Such peaks play a dominating role in the deeper valencestate region (e.g., $\mathrm{E}<-3 \mathrm{eV}$ ) and the opposite region. Some of them merge with the $\left(2 \mathrm{~s}, 2 \mathrm{p}_{x}, 2 \mathrm{p}_{y}, 2 \mathrm{p}_{z}\right)$ peak of the $\mathrm{O}$ atoms, clearly illustrating the significance of the Ge-O bonding in the distorted structure. Shortly, the Li-O and Ge-O chemical bonds are deduced to have multi-orbital hybridizations of $2 \mathrm{~s}-\left(2 \mathrm{p}_{x}, 2 \mathrm{p}_{y}\right.$, $\left.2 \mathrm{p}_{z}\right)$ and $\left(4 \mathrm{~s}, 4 \mathrm{p}_{x}, 4 \mathrm{p}_{y}, 4 \mathrm{p}_{z}\right)-\left(2 \mathrm{~s}, 2 \mathrm{p}_{x}, 2 \mathrm{p}_{y}, 2 \mathrm{p}_{z}\right)$, respectively. This unusual characteristic is strongly consistent with the spatial charge density analysis. As far as we can tell, the existence of the complicated multi-orbital hybridization implies that the $\mathrm{Li}_{2} \mathrm{GeO}_{3}$ band structure is very difficult to simulate by the tightbinding model.

STS measurements (Niimi et al., 2007), an extension of STM, can be efficiency used to examine the number, form, energy and intensity of special Van-hove singularities in DOS. This powerful method has been successfully identified the diverse electronic properties in GNRs (Odom et al., 1998; Huang et al., 2012), carbon nanotubes, graphene (Klusek, 1999)and few-layer graphenes (Li et al., 2010; Luican et al., 2011). For example, the square-root relation peaks in $1 \mathrm{D}$ carbon nanotubes and GNRs (Odom et al., 1998), a symmetric V-shape structure for monolayer graphene (Luican et al., 2011) and finite DOS at Femi level in the AB graphite stacking (Klusek, 1999) have all been investigated. The main features of the electronic properties in the $3 \mathrm{D}$ ternary $\mathrm{Li}_{2} \mathrm{GeO}_{3}$ compound, including the large energy spacing between the occupied and unoccupied bands, the plethora of asymmetric/symmetric peaks, the highly asymmetric electron and hole energy spectra, as well as their widths, could be further investigated with STS. The theoretical predictions of Van Hove singularities, together with the STS measurements are worthy to be identified for the understanding of the complicated multi-orbital hybridizations in the $\mathrm{Li}-\mathrm{O}$ and $\mathrm{Ge}-\mathrm{O}$ bonds, and thus the electronic properties of $\mathrm{Li}_{2} \mathrm{GeO}_{3}$ compound.

Up to now, LIBs have been quickly developed, owing to their vital importance. Their safety issues can be eliminated by changing the liquid to a solid-state electrolyte. The 3D ternary $\mathrm{Li}_{2} \mathrm{GeO}_{3}$ can be adopted to manufacture batteries that are low-cost, durable, and suitable for high-capacity energy storage. Based on the electronic band structure with/without atomic domination, spatial charge distribution, and atom-/orbital-projected DOS calculations, the critical multi-orbital hybridization of $\mathrm{Li}-\mathrm{O}$ and $\mathrm{Ge}-\mathrm{O}$ bonds is obtained. Apparently, the $\mathrm{Li}_{x} \mathrm{Ge}_{y} \mathrm{O}_{z}$ compound has a certain metastable configuration; during battery operation the transformation of many metastable structures (e.g., $\mathrm{Li}_{2} \mathrm{GeO}_{3}, \mathrm{Li}_{4} \mathrm{GeO}_{4}, \mathrm{Li}_{2} \mathrm{Ge}_{7} \mathrm{O}_{15}$ ) occurs. Therefore, further systematic studies on other metastable structures are necessary to identify the most prominent evolution paths.

Generally speaking, there exist various chemical bonds in a large unit cell, a complicated energy sub-band with a large band gap, $\mathrm{E}_{g}=3.77 \mathrm{eV}$, heterogeneous spatial charge densities in diverse chemical bonds, and multi-orbital hybridizations in atom-/orbital-projected DOS. The first-principles electronic structures might be too complicated to be simulated by the phenomenological models. To be specific, the extremely 
non-uniform chemical and physical environments, which survive in the large unit cells of the $3 \mathrm{D}$ ternary $\mathrm{Li}_{2} \mathrm{GeO}_{3}$ compounds, are responsible for the featured electronic structures. Such critical factors cover a lot of multi-orbital hybridizations in the Li-O and $\mathrm{Ge}-\mathrm{O}$ bondings. The orbital-induced various hopping integral site energies should be included in the significant Hamiltonians simultaneously. Apparently, many parameters will be required to get a good fit. It would be very difficult to create a good Hamiltonian diagonalization, and thus, it is very challenging to achieve a concise physical picture for a full understanding of the featured electronic energy spectra.

\section{CONCLUDING REMARKS}

The unusual and unique geometric, electronic properties of the $3 \mathrm{D}$ ternary $\mathrm{Li}_{2} \mathrm{GeO}_{3}$ compound, a potential electrolyte candidate for $\mathrm{Li}^{+}$-based batteries, are investigated by using first principle calculations. The delicate analysis on atom-dominated energy band structure, the charge density distribution in the modulate of the chemical bonds, the atom-/orbital-projected DOS are successfully identified the multi-orbital hybridization in Li-O and Ge-O bonds. This theoretical framework could be generalized for other electrolytic, cathode and anode materials of $\mathrm{Li}^{+}$-based batteries. Furthermore, the extremely non-uniform environment with highly isotropic characteristics in the large unit cell poses a great challenge for the tightbinding model.

In the current work, the solid-state electrolyte material $\mathrm{Li}_{2} \mathrm{GeO}_{3}$, with 24 atoms in a large computational cell is an orthorhombic crystal structure. There exist $32 \mathrm{Li}-\mathrm{O}$ and $16 \mathrm{Ge}-$ $\mathrm{O}$ chemical bonds, in which each $\mathrm{Li} / \mathrm{Ge}$ atom is surrounded by four $\mathrm{O}$ atoms in the tetrahedral form. Most importantly, the strong covalent bondings create a huge indirect energy gap of $\mathrm{E}_{g}=3.77 \mathrm{eV}$. The band structure lying in range -6.5 to $6.5 \mathrm{eV}$ is strong relative to the critical chemical bondings. There exist many sub-bands with high anisotropy, strong energy dispersion and frequently anti-crossing/crossing/non-crossing phenomena, and a strong contradistinction of electron and hole states appears near the Fermi level. The band structure with atomic domination, spatial charge density and the atom-/orbital-projected DOS are

\section{REFERENCES}

Balog, R., Jrgensen, B., Nilsson, L., Andersen, M., Rienks, E., Bianchi, M., et al. (2010). Bandgap opening in graphene induced by patterned hydrogen adsorption. Nat. Mater. 9, 315-319. doi: 10.1038/nmat2710

Carstens, T., Ispas, A., Borisenko, N., Atkin, R., Bund, A., and Endres, F. (2016). In situ scanning tunneling microscopy (STM), atomic force microscopy (AFM) and quartz crystal microbalance (EQCM) studies of the electrochemical deposition of tantalum in two different ionic liquids with the 1-butyl-1-methylpyrrolidinium cation. Electrochim. Acta 197, 374-387. doi: 10.1016/j.electacta.2015.07.178

Chen, H., Dawsona, J. A., and Hardinga, J. H. (2014). Effects of cationic substitution on structural defects in layered cathode materials LiNiO2. J. Mater. Chem. A 2, 7988-7996. doi: 10.1039/C4TA00637B

Chen, R., Qu, W., Guo, X., Li, L., and Wu, F. (2016). The pursuit of solid-state electrolytes for lithium batteries: from comprehensive insight successfully identify the important multi-orbital $2 \mathrm{~s}-\left(2 \mathrm{p}_{x}, 2 \mathrm{p}_{y}\right.$, $\left.2 \mathrm{p}_{z}\right)$ and $\left(4 \mathrm{~s}, 4 \mathrm{p}_{x}, 4 \mathrm{p}_{y}, 4 \mathrm{p}_{z}\right)-\left(2 \mathrm{~s}, 2 \mathrm{p}_{x}, 2 \mathrm{p}_{y}, 2 \mathrm{p}_{z}\right)$ hybridizations. The theoretical predictions on the structural relaxation are verified by PXRD, TEM, and SEM, while the band gap, the occupied electronic states and the special Van-Hove singularities could be examined by optical spectroscopy, ARPES and STS measurements, respectively.

The calculated results clearly illustrate that the 3D ternary $\mathrm{Li}_{2} \mathrm{GeO}_{3}$ compound could be used as a solid-state electrolyte. During the battery operation, the structural transformation between the current meta-stable structure with other metastable structures are expected to occur at any time. This study is able to provide certain meaningful information about the critical mechanisms in the ion transport. It might be helpful to solve the open issue: the most optimal evolution paths of the $\mathrm{Li}^{+}$migration during the discharging processes of the all solid-states LIBs.

\section{DATA AVAILABILITY STATEMENT}

The raw data supporting the conclusions of this article will be made available by the authors, without undue reservation.

\section{AUTHOR CONTRIBUTIONS}

VK: conceptualization, model building, methodology, software, validation, writing- reviewing, and editing. NT, TN, TH, and HP: model building, data curation, visualization, and original draft preparation. M-FL: conceptualization, model building, methodology, software, validation, reviewing and editing, and supervision.

\section{FUNDING}

This work was supported by the Hierarchical Green Energy Materials (Hi-GEM) Research Center, from The Featured Areas Research Center Program within the framework of the Higher Education Sprout Project by the Ministry of Education (MOE) and the Ministry of Science and Technology (MOST 108- 3017F-006 -003) in Taiwan. to emerging horizons. Mater. Horizons 3, 487-516. doi: 10.1039/C6MH0 $0218 \mathrm{H}$

Cheng, F., Liang, J., Tao, Z., and Chen, J. (2011). Functional materials for rechargeable batteries. Adv. Mater. 23, 1695-1715. doi: 10.1002/adma.201003587

Cheng, F., Tao, Z., Liang, J., and Chen, J. (2008). Template-directed materials for rechargeable lithium-ion batteries. Chem. Mater. 20, 667-681. doi: $10.1021 / \mathrm{cm} 702091 \mathrm{q}$

Coletti, C., Forti, S., Principi, A., Emtsev, K. V., Zakharov, A. A., Daniels, K. M., et al. (2013). Revealing the electronic band structure of trilayer graphene on SiC: an angle-resolved photoemission study. Phys. Rev. B 88:155439. doi: 10.1103/PhysRevB.88.155439

Emly, A., Kioupakis, E., and Van der Ven, A. (2013). Phase stability and transport mechanisms in antiperovskite $\mathrm{Li}_{3} \mathrm{OCl}$ and $\mathrm{Li}_{3} \mathrm{OBr}$ superionic conductors. Chem. Mater. 25, 4663-4670. doi: 10.1021/cm40 16222 
Feist, A., Bach, N., da Silva, N. R., Danz, T., Moller, M., Priebe, K. E., et al. (2017) Ultrafast transmission electron microscopy using a laser-driven field emitter: femtosecond resolution with a high coherence electron beam. Ultramicroscopy 176, 63-73. doi: 10.1016/j.ultramic.2016.12.005

Furusawa, S., and Enokida, S. (2011). Ionic conductivity of polycrystalline $\mathrm{Li} 2 \mathrm{Ge}_{x} \mathrm{Si}_{1-x} \mathrm{O}_{3}(x=0.0 \sim 1.0)$. Key Eng. Mater. 459, 27-31. doi: 10.4028/www.scientific.net/kem.459.27

Gruneis, A., Attaccalite, C., Pichler, T., Zabolotnyy, V., Shiozawa, H., Molodtsov, S., et al. (2008). Electron-electron correlation in graphite: a combined angle-resolved photoemission and first-principles study. Phys. Rev. Lett. 100:037601. doi: 10.1103/PhysRevLett.100. 037601

Hansma, P. K. (1987). Scanning tunneling microscopy. J. Appl. Phys. 61, 1-24. doi: $10.1063 / 1.338189$

Haussuuhl, S., Liebertz, J., and Stahr, S. (1982). Single crystal growth and pyroelectric, dielectric, piezoelectric, elastic, and thermoelastic properties of orthorhombic $\mathrm{Li}_{2} \mathrm{SiO}_{3}, \mathrm{Li}_{2} \mathrm{GeO}_{3}$, and $\mathrm{Na}_{2} \mathrm{GeO}_{3}$. Crystal Res. Technol. (1982) 17, 521-526. doi: 10.1002/crat.2170170421

Holder, C. F., and Schaak, R. E. (2019). Tutorial on powder x-ray diffraction for characterizing nanoscale materials. ACS Nano. 13, 7359-7365. doi: 10.1021/acsnano.9b05157

Huang, H., Wei, D., Sun, J., Wong, S. L., Feng, Y. P., Neto, A. H. C., et al. (2012). Spatially resolved electronic structures of atomically precise armchair graphene nanoribbons. Sci. Rep. 2:983. doi: 10.1038/srep00983

Kaboudvand, F., Vinckeviciute, J., Kolli, S., Radin, M. D., and Van der Ven, A. (2019) Phase stability and electronic structure of tin sulfide compounds for Liion batteries. J. Phys. Chem. C 123, 29086-29095. doi: 10.1021/acs.jpcc.9b06902

Kataura, H., Kumazawa, Y., Maniwa, Y., Umezu, I., Suzuki, S., Ohtsuka, Y., and Achiba, Y. (1999). Optical properties of single-wall carbon nanotubes. Synthet. Metals 103, 2555-2558. doi: 10.1016/S0379-6779(98)00278-1

Kelly, K. F., Chiang, I. W., Mickelson, E. T., Hauge, R. H., Margrave, J. L., Wang, X., et al. (1999). Insight into the mechanism of sidewall functionalization of single-walled nanotubes: an STM study. Chem. Phys. Lett. 313, 445-450. doi: 10.1016/S0009-2614(99)00973-2

Kim, K., Walter, A., Moreschini, L., Seyller, T., Horn, K., Rotenberg, E., et al. (2013). Coexisting massive and massless Dirac fermions in symmetry-broken bilayer graphene. Nat. Mater. 12, 887-892. doi: 10.1038/nmat3717

Klusek, Z. (1999). Investigations of splitting of the $\pi$ bands in graphite by scanning tunneling spectroscopy. Appl. Surface Sci. 151, 251-261. doi: 10.1016/S0169-4332(99)00282-2

Kresse, G., and Hafner, J. (1993). Ab initio molecular dynamics for liquid metals. Phys. Rev. B 47, 558-561. doi: 10.1039/C5CP04841A

Kresse, G., and Joubert, D. (1999). From ultrasoft pseudopotentials to the projector augmented-wave method. Phys. Rev. B 59, 1758-1775. doi: 10.1103/PhysRevB.59.1758

Kuganathan, N., Tsoukalas, L. H., and Chroneos, A. (2019). Defects, dopants and Li-ion diffusion in $\mathrm{Li}_{2} \mathrm{SiO}_{3}$. Solid State Ionics 335, 61-66. doi: 10.1016/j.ssi.2019.02.019

Lee, J. K., Lee, S. C., Ahn, J. P., Kim, S. C., Wilson, J. I., and John, P. (2008). The growth of AA graphite on (111) diamond. J. Chem. Phys. (2008) 129:234709. doi: $10.1063 / 1.2975333$

Li G, Luican A, Dos Santos J L, Neto A C, Reina A, Kong J, and Andrei, E Y. Observation of Van Hove singularities in twisted graphene layers. Nature Physics. (2010) 6:109. doi: 10.1038/nphys1463

Liebert, B. E., and Huggins, R. A. (1976). Ionic conductivity of $\mathrm{Li}_{4} \mathrm{GeO}_{4}, \mathrm{Li}_{2} \mathrm{GeO}_{3}$ and $\mathrm{Li}_{2} \mathrm{Ge}_{7} \mathrm{O}_{15}$. Mater. Res. Bull. 11, 533-538. doi: 10.1016/0025-5408(76)90235-X

Lin, S.-Y., Chang, S.-L., Tran, N. T. T., Yang, P.-H., and Lin, M.-F. (2015a). H-Si bonding-induced unusual electronic properties of silicene: a method to identify hydrogen concentration. Phys. Chem. Chem. Phys. 17, 26443-26450.

Lin, Y. T., Chung, H. C., Yang, P.-H., Lin, S.-Y., and Lin, M.-F. (2015b). Adatom bond-induced geometric and electronic properties of passivated armchair graphene nanoribbons. Phys. Chem. Chem. Phys. 17, 16545-16552. doi: 10.1039/C5CP02226F

Liu, L., Wang, Z., Li, H., Chen, L., and Huang, X. (2002). Al2O3-coated LiCoO2 as cathode material for lithium ion batteries. Solid State Ionics 152-153, 341-346. doi: 10.1016/S0167-2738(02)00333-8
Lu, D., Vishik, I. M., Yi, M., Chen, Y., Moore, R. G., and Shen, Z. X. (2012). Angleresolved photoemission studies of quantum materials. Annu. Rev. Cond. Matter Phys. 3, 129-167. doi: 10.1146/annurev-conmatphys-020911-125027

Lu, X., Howard, J. W., Chen, A., Zhu, J., Li, S., Wu, G., et al. (2016). Antiperovskite $\mathrm{Li}_{3} \mathrm{OCl}$ superionic conductor films for solid-state Li-ion batteries. Adv. Sci. 3:1500359. doi: 10.1002/advs.201500359

Lu, Z., Chen, C., Baiyee, Z. M., Chen, X., Niub, C., and Ciuccia, F. (2015). Defect chemistry and lithium transport in $\mathrm{Li}_{3} \mathrm{OCl}$ anti-perovskite superionic conductor. Phys. Chem. Chem. Phys. 17, 32547-32555. doi: 10.1039/C5CP05722A

Luican, A., Li, G., Reina, A., Kong, J., Nair, R., Novoselov, K. S., et al. (2011). Singlelayer behavior and its breakdown in twisted graphene layers. Phys. Rev. Lett. 106:126802. doi: 10.1103/PhysRevLett.106.126802

Maitra, U., Matte, H. S. S., Kumar, P., and Rao, C. N. R. (2012). Strategies for the synthesis of graphene, graphene nanoribbons, nanoscrolls and related materials. Chim. Int. J. Chem. 66, 941-948. doi: 10.2533/chimia.2012.941

Nakagawa, A., Kuwata, N., Matsuda, Y., and Kawamura, J. (2010). Characterization of stable solid electrolyte Lithium Silicate for thin film Lithium battery. Phys. Soc. Japan 79, 98-100. doi: 10.1143/JPSJS.79SA.98

Nguyen, T. D. H., Pham, H. D., Linb, S. Y., and Lin, M. F. (2020). Featured properties of $\mathrm{Li}^{+}$-based battery anode: $\mathrm{Li}_{4} \mathrm{Ti}_{5} \mathrm{O}_{12}$. RSC Adv. 10, 14071-14079. doi: 10.1039/D0RA00818D

Niimi, Y., Kanisawa, K., Kojima, H., Kambara, H., Hirayama, Y., Tarucha, S., et al. (2007). STM/STS measurements of two-dimensional electronic states in magnetic fields at epitaxially grown InAs(111)A surfaces. J. Phys. Conf. Ser. 61:174. doi: 10.1088/1742-6596/61/1/174

Odom, T. W., Huang, J. L., Kim, P., and Lieber, C. M. (1998). Atomic structure and electronic properties of single-walled carbon nanotubes. Nature 391, 62-64. doi: $10.1038 / 34145$

Ohta, T., Bostwick, A., McChesney, J. L., Seyller, T., Horn, K., and Rotenberg, E. (2007). Interlayer interaction and electronic screening in multilayer graphene investigated with angle-resolved photoemission spectroscopy. Phys. Rev. Lett. 98:206802. doi: 10.1103/PhysRevLett.98.206802

Perdew, J. P., Burke, K., and Ernzerhof, M. (1996). Generalized gradient approximation made simple. Phys. Rev. Lett. 77, 3865-3868. doi: 10.1103/PhysRevLett.77.3865

Rahman, M. M., Sultana, I., Yang, T., Chen, Z., Sharma, N., Glushenkov, A. M., et al. (2016). Lithium germanate $\left(\mathrm{Li}_{2} \mathrm{GeO}_{3}\right)$ : a high-performance anode material for lithium-ion batteries. Angew. Chem. Int. Ed. 55, 16059-16063. doi: 10.1002/anie.201609343

Rincon, C., Wasim, S. M., Martin, G., and Snchez Perez, G. (2003). Optical absorption spectra near the fundamental band edge in $\mathrm{Cu}_{2} \mathrm{In}_{4} \mathrm{Se}_{7} \mathrm{Cu}_{2} \mathrm{In}_{4} \mathrm{Se}_{7}$ bulk crystals. J. Appl. Phys. (2003) 93, 8939-8944. doi: 10.1063/1.15 67800

Sakurai, Y., Arai, H., Okada, S., and Yamaki, J. (1997). Low temperature synthesis and electrochemical characteristics of $\mathrm{LiFeO} 2$ cathodes. J. Power Sour. 68, 711-715. doi: 10.1016/S0378-7753(96)02579-7

Tarascon, J.-M., and Armand, M. (2001). Issues and challenges facing rechargeable lithium batteries. Nature 414, 359-367. doi: 10.1038/35104644

Tran, N. T. T., Lin, S.Y., Lin, C.Y., and Lin, M. F. (2018). Geometric and Electronic Properties of Graphene-Related Systems. Boca Raton, FL: CRC Press. doi: $10.1201 / \mathrm{b} 22450$

Trukhin, A. N., Rogulis, U., and Spingis, M. (1997). J. Luminescence 72-74, 890-892. doi: 10.1016/S0022-2313(96)00418-8

Van der Lit, J., Jacobse, P. H., Vanmaekelbergh, D., and Swart, I. (2015). Bending and buckling of narrow armchair graphene nanoribbons via STM manipulation. N. J. Phys. 17:053013. doi: 10.1088/1367-2630/17/5/ 053013

Wang, Y., Richards, W., Ong, S., Miara, L. J., Kim, J. C., Mo, Y., et al. (2015). Design principles for solid-state lithium superionic conductors. Nat. Mater. 14, 1026-1031. doi: 10.1038/nmat4369

Wang, Y., Song, S., Xu, C., Hu, N., Molenda, J., and Lu, L. (2019). Development of solid-state electrolytes for sodium-ion battery-A short review. Nano Mater. Sci. 1, 91-100. doi: 10.1016/j.nanoms.2019.02.007

Warner, J. H., Rummeli, M. H., Gemming, T., Buchner, B., and Briggs, G. A. D. (2008). Direct imaging of rotational stacking faults in few layer graphene. Nano Lett. 9, 102-106. doi: 10.1021/nl8025949 
Wisesa, P., McGill, K. A., and Mueller, T. (2016). Efficient generation of generalized Monkhorst-Pack grids through the use of informatics. Phys. Rev. B 93:155109. doi: 10.1103/PhysRevB.93.155109

Wu, M., Xu, B., Lei, X., Huang, K., and Ouyang, C. (2018). Bulk properties and transport mechanisms of a solid state antiperovskite $\mathrm{Li}$-ion conductor $\mathrm{Li}_{3} \mathrm{OCl}$ : insights from first principles calculations. J. Mater. Chem. A 6, 1150-1160. doi: 10.1039/C7TA08780B

Xiang, H. F., Li, Z. D., Xie, K., Jiang, J. Z., Chen, J. J., Lian, P. C., et al. (2012). Graphene sheets as anode materials for Li-ion batteries: preparation, structure, electrochemical properties and mechanism for lithium storage. RSC Adv. 2, 6792-6799. doi: 10.1039/C2RA20549A

Xiao, R., Li, H., and Chen, L. (2015). Candidate structures for inorganic Lithium solid-state electrolytes identified by high-throughput bond-valence calculations. J. Materiomics 1, 325-332. doi: 10.1016/j.jmat.2015.08.001

Yin C, Xiang H, Li C, Porwal H, Fang L. (2018). Low-temperature sintering and thermal stability of $\mathrm{Li}_{2} \mathrm{GeO}_{3}$-based microwave dielectric ceramics with low permittivity. J Am Ceram Soc. (2018) 101: 4608-4614. doi: 10.1111/jace.15723

Yu, Y. S., Prabhu, S. S., Perkowitz, S., and Kim, S. C. (1987). Polariton modes and materials parameters in $\mathrm{Li}_{2} \mathrm{GeO}_{3}$. Phys. Rev. B 56, 5046-5048. doi: 10.1103/PhysRevB.56.5046

Zhang, J., Xiao, J., Meng, X., Monroe, C., Huang, Y., and Zuo, J. M. (2010). Free folding of suspended graphene sheets by random mechanical stimulation. Phys. Rev. Lett. (2010) 104:166805. doi: 10.1103/PhysRevLett.104.166805

Zhang, Y., Zhao, Y., and Chen, C. (2013). Ab initio study of the stabilities of and mechanism of superionic transport in lithium-rich antiperovskites. Phys. Rev. B 87:134303. doi: 10.1103/PhysRevB.87.1 34303

Zhao, G., Suzuki, K., Yonemura, M., Hirayama, M., and Kanno, R. (2019). Enhancing fast Lithium ion conduction in $\mathrm{Li}_{4} \mathrm{GeO}_{4}-\mathrm{Li}_{3} \mathrm{PO}_{4}$ solid electrolytes. ACS Appl. Energy Mater. 2, 6608-6615. doi: 10.1021/acsaem.9b 01152

Zhao, Q., Stalin, S., Zhao, C. Z., and Archer, L. A. (2020). Designing solidstate electrolytes for safe, energy-dense batteries. Nat. Rev. Mater. 5, 229-252. doi: 10.1038/s41578-019-0165-5

Zulueta, Y. A., Nguyen, M. T., and Dawson, J. A. (2020). Na- and KDoped $\mathrm{Li} 2 \mathrm{SiO} 3$ as an alternative solid electrolyte for solid-state Lithium batteries. J. Phys. Chem. C 124, 4982-4988. doi: 10.1021/acs.jpcc.9b 10003

Conflict of Interest: The authors declare that the research was conducted in the absence of any commercial or financial relationships that could be construed as a potential conflict of interest.

Copyright $\odot 2020$ Khuong Dien, Thi Han, Nguyen, Huynh, Pham and Lin. This is an open-access article distributed under the terms of the Creative Commons Attribution License (CC BY). The use, distribution or reproduction in other forums is permitted, provided the original author(s) and the copyright owner(s) are credited and that the original publication in this journal is cited, in accordance with accepted academic practice. No use, distribution or reproduction is permitted which does not comply with these terms. 